\title{
Neue Perspektiven in der Bildung angehender Fremdsprachenlehrer ${ }^{1}$
}

\author{
New perspectives on education of foreign \\ language teachers
}

\begin{abstract}
The aim of the following article is to present the newest research on philology students concerning their professional perspectives and needs. This paper outlines some important aspects of education referring to the foreign language teachers and their preparation for the future work like developing practical teaching skills and work with different age groups.
\end{abstract}

KEYWORDS: foreign language teacher, work, senior group, age-diversified learners, work expectations.

\section{GERAGOGIK ALS EIN ZUKUNFTSTRÄCHTIGER WISSENSCHAFTSZWEIG}

Geragogik wird als „eine junge und in mancher Hinsicht gerade erst entstehende Wissenschaft" (Kolland 2010: 9) beschrieben. Um dieses neue gesellschaftlich- wissenschaftliche Phänomen besser verstehen zu können, ist es angebracht sich mit den Rahmenbedingungen moderner Geragogik vertraut zu machen, die auch im Bereich der fremdsprachlichen Geragogik gelten und im Zentrum des Interesses des vorliegenden Artikels stehen. Kolland (2010: 10ff) schlägt eine gründliche Analyse dreier Rahmenbedingungen vor, die den geragogischen Prozessen zugrunde liegen. Darunter

${ }^{1}$ Um den Lesefluss nicht zu stören, wurde in dieser Arbeit bei den Personenbezeichnungen auf geschlechtsspezifische Unterscheidungen verzichtet und die gebräuchlichere maskuline Form gewählt, die für Personen beiderlei Geschlechts gleichermaßen gilt. 
werden: der demografische Wandel, die Entstehung einer neuen Lebensphase - Alters, sowie die Expansion des Bildungs- und Wissenssystems genannt. Die erste Bedingung des demografischen Wandels, ist auf die Verlängerung der Lebenserwartung zurückzuweisen. Dies ermöglicht neue Chancen vor allem im Bereich der eignen Entwicklung und des lebenslangen Lernens. Das längere Leben gibt den Menschen mehr Zeit für die Selbstentfaltung, die im kontinuierlichen Kontext verstanden wird. Die zweite Lebensbedingung bezieht sich auf die Aussonderung der neuen Lebensphase - des Alters und zwar des aktiven Alters. In Bezug auf diese Zeit werden neue Erwartungen gestellt, die vorher nicht berücksichtigt worden sind. Die heutigen Senioren verlangen mehr vom Leben, wollen immer aktiver sein. Keller (2009: 63) betont die Entstehung der neuen Lebensphase, die „einen eigenen bildungstheoretischen und bildungspraktischen Zugang verlangt." Die dritte Rahmenbedingung bezieht sich auf die fortschreitende Expansion des Bildungsund Wissenssystems. Ohne Bildung ist die Entwicklung kaum vorstellbar, deswegen wächst in der Epoche der wachsenden Selbstentwicklungsmöglichkeiten das Interesse an Bildung auch in der Gruppe der Senioren.

Diese drei Rahmenbedingungen stellen drei Säulen der Geragogik dar, die als Wissenschaftszweig von Veelken (2000: 88) folgend definiert wird: "Geragagogik hat es zu tun mit Lebensbewältigung, Lebensgestaltung, Selbstverwirklichung, Empowerment. Es geht dabei sowohl um die Entfaltung der Identität im Sozialisationsprozess als auch um kritische Analyse und Veränderung vorgegebener Sozialisationsagenturen und mit ihnen verbundenen Rollenmuster."

Bubolz-Lutz und andere (2010: 14) weisen zudem darauf hin, dass der Gegenstandsbereich der Geragogik zweipolig in der Fachliteratur behandelt wird. Einerseits wird das Lernen im Alter hervorgehoben, andererseits wird sich auf die Bildungsarbeit der Lehrkräfte mit alten Menschen fokussiert. In dem vorliegenden Artikel wird auf den zweiten Aspekt aufmerksam gemacht, d.h. wie die angehenden Lehrer auf die Arbeit mit den Senioren vorbereitet werden und welche Bedürfnisse in dieser Hinsicht in der Hochschulbildung entstehen.

\section{LEHRSTUDIENGÄNGE AN DEN POLNISCHEN UNIVERSITÄTEN}

Lehrstudiengänge umfassen nicht nur ein breites Spektrum verschiedener Veranstaltungen, wie Psychologie, Pädagogik, Stimmeemission, im Falle der angehenden Fremdsprachenlehrer ist im Lehrkurrikulum auch Fremdsprachendidaktik und -methodik zu finden. Diese Lehrveranstaltungen entspre- 
chen den ministeriellen Rahmen und bereiten gleichzeitig die Studenten auf ihre künftige Arbeit vor. Zusätzlich sind die Studenten verpflichtet, neben dem sehr umfangreichen Stundenaufwand an der Universität Praktika in den Grund- und weiterführenden Schulen zu absolvieren, alles, um ihre beruflichen Perspektiven zu erweitern und ihren baldigen Arbeitspflichten besser nachzugehen. Diese Vorschläge und Bemühungen einzelner Fakultäten finden einen Niederschlag darin, dass ihre Absolventen tatsächlich immer komplexer für den Lehrerberuf vorbereitet sind, obwohl es laut Untersuchung von Wiłkomirska (2005) an praktischer Vorbereitung junger Lehrer auf die authentische Arbeit in der Schule fehlt, was die befragten Schulleiter betonnen.

Die Tatsache, dass die Altersstruktur der potenziellen Lerner sich in der letzten Zeit dramatisch geändert hat, beeinflusst stark die Erfordernisse des Arbeitsmarkts und dadurch auch die Situation und Perspektiven der Absolventen der Lehrstudiengänge, darunter neuphilologischer Studienrichtungen. Im weiteren Teil des Artikels wird gerade auf die oben genannten Aspekte hingewiesen. Die in dem vorliegenden Artikel dargestellten Schlussfolgerungen sind das Ergebnis der von der Autorin durchgeführten Umfrage unter den Studenten der Angewandten Linguistik an der Adam-Mickiewicz-Universität in Poznań. Die durchgeführte Umfrage wurde nach dem Prinzip: Datenerhebung, Datengewinnung und Datenauswertung (vgl. Caspari, Helbig, Schmelter 2003: 501ff) entworfen.

\section{DATENERHEBUNG - ZIELGRUPPE}

Am 26. und 27. März 2012 fand im Institut der Angewandten Linguistik eine zyklische Minikonferenz unter dem Titel: „Glottodidaktik- gestern, heute und morgen" statt. Die Studenten (vor allem angehende Lehrer) nahmen an verschiedenen Vorlesungen und Veranstaltungen teil, darunter gab es einen Workshop zum Thema: „Neue Perspektiven und neue Altersgruppen im Lehrerberuf". Während der Veranstaltung wurden die neusten Methoden und Techniken der Arbeit im Fremdsprachenunterricht mit neuen Altersgruppen, dabei auch mit Senioren, präsentiert. Vor dem Beginn des Workshops wurden die Studenten gebeten, an einer kurzen Umfrage teilzunehmen. Diese Umfrage diente dem Ziel, einerseits die Orientierung der Studenten zum Thema Geragogik und Fremdsprachengeragogik zu erkennen, andererseits ihre Erwartungen und Bedürfnisse auf die Vorbereitung zur Arbeit mit der neuen Lernergruppe kennenzulernen. Neben den festgestellten Zielen der Umfrage wurden auch zusätzliche gesammelt, unter anderem, wie sich die Studenten ihre zukünftige Arbeit vorstellen oder wovor sie im beruflichen Kontext Angst haben. 


\subsection{Fragenstruktur}

Die Umfrage beginnt mit einer kurzen Einleitung, in der die Befragten nach dem Geschlecht und Studienjahr gefragt wurden. Danach folgen die tatsächlichen Fragen, die der Meinungsforschung dienen. Die Umfrage besteht aus acht Fragen, die sowohl einen geschlossenen als auch offenen Charakter haben. Die Fragen werden in zwei Gruppen eingeteilt. Zur ersten Gruppe gehören die Fragen, die die Orientierung der Studenten und ihr Wissen im Bereich der Fremdsprachengeragogik erörtern. Die zweite Gruppe stellen die Fragen dar, die die Einstellung der Studenten zur Arbeit mit der neuen Lernergruppe- Senioren und ihre Erwartungen an die Studienlehrprogramme betreffen. Die offenen Fragen ermöglichen den Studenten sich frei zu den besagten Themen zu äußern, was als zusätzliche Informationsquelle dient.

\subsection{Datenauswertung}

Die Datenerhebung wird durch die Datenauswertung gefolgt. Die Rolle der Datenauswertung ist es die gesammelten Daten nach einem Schlüssel zu ordnen und sie einer Analyse zu unterziehen (vgl. Caspari, Helbig, Schmelter 2003: 501ff). Die in der Umfrage erhobenen Daten werden im weiteren Teil des Beitrags, vor allem nach dem Prinzip der Quantität, verglichen. Die freien oder meinungsbildenden Äußerungen werden separat qualitativ betrachtet.

\subsection{Geschlechtsstruktur der Befragten}

Unter 35 Befragten gab es lediglich 3 Männer, was einen Beweis dafür liefert, dass die Lehrstudiengänge vor allem durch Frauen gewählt werden. Dies ist auch ein sehr relevanter Aspekt, der vielleich tieferer Studien und Änderungsentwürfe bedarf, weil sowohl weibliche als auch männliche Lehrkräfte in den Schulen gebraucht werden.

\section{DIE ERSTE FRAGENGRUPPE}

Die erste Fragengruppe bezieht sich auf die Orientierung der Studenten in den geragogischen Schwerpunkten. Zu den grundlegenden Begriffen der Geragogik gehört die Definition des Wortes Senior. In der Fachliteratur herrscht bisher keine Einigkeit, wer zu der Seniorengruppe gehören sollte. 
Umso interessanter sind somit die Äußerungen der Studenten, die ihre Meinungen auf dem allgemeinen Wissen, manchmal auch auf einigen Stereotypen aufbauen.

Am häufigsten (19 Befragten) wiederholte sich die Antwort, dass zur Seniorengruppe „ältere Personen" gehören, ohne eine genaue Altersgrenze anzugeben. In anderen Fragebögen waren konkrete zeitliche Grenzen gesetzt. Die erhobenen Ergebnisse werden in den unteren Tabelle dargestellt.

Die Anzahl der Befragten

1

2

1

7

2

3
Die Altersgrenze ab der man die Zugehörigkeit zur Seniorengruppe verzeichnet

$50+$

$55+$

nach dem 50-60 Lebensjahr

$60+$

$65+$

$70+$

In 10 Antworten tauchte auch die Ergänzung auf, dass „diese Personen (Senioren), nicht mehr berufstätig sind $2^{\prime \prime}$. Eine Person betonte in der Antwort auch den Aspekt der Freizeit: "Senior- to osoba w starszym wieku, która ma bardzo dużo wolnego czasu. Senior - eine Person im höheren Alter, die über viel Freizeit verfügt". In 2 Antworten wurde auch, neben dem Alter, die Erfahrung als das Gruppenzugehörigkeitskriterium genannt. 2 Personen berücksichtigten in ihren Antworten auch die aktive Dimension dieser Lebensphase.

Beachtenswert ist, dass in fast allen Antworten die älteren Personen neutral dargestellt wurden, es gab keine Antwort, in der die Senioren im negativen Licht gestellt wurden. Nur in einem Fragebogen lässt sich eine sehr positive Darstellung älterer Personen verzeichnen: "Senior - to osoba starsza, cały czas uśmiechnięta, z dużym bagażem doświadczenia. Senior eine ältere Person, Strahlemann, mit großem Erfahrungsschatz."

In der zweiten Frage dieses Fragenkomplexes wurden die Teilnehmer gebeten zu entscheiden, ob und in wie fern die Seniorengruppe sich von anderen Lernergruppen unterschiedet. Dabei herrschte Einigkeit unter den Befragten, weil alle auf Unterschiede zwischen den Gruppen hingedeutet haben. Nur 12 Befragte waren imstande die Unterschiede zu nennen. Zu den auffälligsten Unterschieden gehören, laut den Befragten, ein langsameres Tempo der Arbeit, frühere Erfahrungen mit dem Fremdsprachenlernen, alte Gewohnheiten.

\footnotetext{
${ }^{2}$ Alle Aussagen wurden von der Autorin des Artikels ins Deutsche übersetzt.
} 
Die nächste Frage betrifft die Vorstellung der Befragten über die Größe und die Bedeutung der Seniorengruppe im Kontext des Lehrens. Die Mehrheit der Befragten (17 Personen) bestätigt, dass die Seniorenlerner tatsächlich eine zahlreiche und immer wichtigere Gruppe im didaktischen Kontext darstellen. 10 Personen äußerten sich negativ und 8 Befragte hatten keine Meinung zu diesem Thema.

34 von 35 Befragten deklarierten, dass sie über keine Erfahrung im Bereich des Unterrichtens älterer Menschen verfügen. Nur eine Person hat schon einige Erfahrungen in der didaktischen Arbeit mit Senioren gesammelt.

\section{DIE ZWEITE FRAGENGRUPPE}

Der zweite Fragenkomplex diente dem Ziel, die Einstellung der angehenden Lehrer im Hinblick auf die didaktische Arbeit mit Senioren und ihre Vorbereitung auf diese Arbeit kennen zu lernen.

5 Studenten vertreten die Meinung, dass sie gut zur didaktischen Arbeit mit verschiedenen Altersgruppen (darunter auch mit Senioren) durch die im Studium angebotenen Veranstaltungen vorbereitet wurden. 30 Befragte weisen auf die mangelhafte Vorbereitung zum Unterrichten altersdifferenzierter Lernergruppen hin.

Alle Befragten sind jedoch der Auffassung, dass es sich lohnt die glottodidaktische Theorie und Praxis zu vertiefen. Bei dieser Frage hatten die Befragten die Möglichkeit ihre Begründung frei zu äußern, was der Autorin des vorliegenden Artikels viele zusätzliche Informationen geliefert hat. Die erhobenen Antworten wurden in folgende Kategorien eingeteilt, die im weiteren Teil des Artikels besprochen wurden:

- Neugier,

- Zukunftsperspektiven,

- Mode,

- zunehmende Seniorengruppe,

- Voraussetzung des Lehrerberufes.

\subsection{Neugier}

10 Befragte äußerten Interesse am Unterrichten der Seniorengruppe aus Neugier. Diese Gruppe ist neu auf dem Bildungsmarkt und stellt darüber hinaus große Herausforderung für Lehrer, denen häufig ausreichende didaktische Materialien zur Verfügung nicht stehen. 
Seniorzy jako grupa to prawdziwe wyzwanie dla nauczycieli języków obcych.

Seniorenlerner stellen eine richtige Herausforderung für Fremdsprachenlehrer dar.

Jestem ciekawa, jak wyglądają rzeczywiste zajęcia z tą grupą wiekową i dlatego chciałabym po pierwsze dowiedzieć się więcej na temat seniorów, a po drugie wykorzystać moją wiedzę także w praktyce.

Ich bin sehr neugierig, wie die Arbeit mit dieser Altersgruppe wirklich im Unterricht aussieht, erstens möchte ich gerne mehr über diese Gruppe erfahren und zweitens mein Wissen in der Praxis einsetzten.

\subsection{Zukunftsperspektiven}

20 Befragte haben in ihren Antworten auf die beruflichen Perspektiven hingewiesen. Die Umfrageteilnehmer haben die Schwierigkeiten auf dem Arbeitsmarkt hervorgehoben, deswegen wollen sie noch während ihres Studiums verschiedene Erfahrungen sammeln, die ein wichtiger Punkt in ihrem künftigen Berufswerdegang sein könnten.

Nie wiem jeszcze, z jaka grupą wiekową będę pracowała w przyszłości.

Ich weiß noch nicht mit welcher Altersgruppe ich in der Zukunft arbeiten werde.

Myślę, że na rynku pracy dodatkowe umiejętności są bardzo ważne.

Ich bin der Meinung, dass auf dem Arbeitsmarkt zusätzliche Fähigkeiten von großer Bedeutung sind.

\subsection{Mode}

Für 7 Befragte ist die steigende Bedeutung der Seniorengruppe im Kontext des Fremdsprachenlernens und Lehrens ein neuer Trend. Dieser Trend entwickelt sich sehr schnell und angehende Lehrer müssen den neusten Markt- und Gesellschafterwartungen entgegenkommen.

Aktywność w starszym wieku należy do rozpowszechniającej się mody, która przybiera na sile. [...] Częścią tego trendu jest nauka języków obcych wśród osób starszych. Uważam, że jest to wystarczający powód, aby zainteresować się tym zjawiskiem.

Aktivität im höheren Alter gehört zur sich verbreitenden Modeerscheinung, die sich immer stärker ausprägt. [...] Fremdsprachenlernen im Alter gehört zu diesem Trend. Ich bin der Meinung, dass das ein ausreichender Grund dafür ist, sich für dieses Phänomen zu interessieren. 


\subsection{Zunehmende Seniorengruppe}

Ganze 20 Befragte betonten, dass die Senioren angesichts der immer älter werdenden Gesellschaft eine große und ständig zunehmende soziale Gruppe darstellen. Diese Tatsache trägt dazu bei, dass Senioren ein großes Interesse der Öffentlichkeit wecken und zu aktiven Teilnehmern des sozialen Lebens werden. Sie wollen auch im Alter Denkhorizonte erweitern und Kenntnisse Vertiefen.

Wcześniej nauka języków obcych nie była tak rozpowszechniona wśród osób starszych, jak to ma miejsce dzisiaj. Myślę, że jest to bezpośredni wynik tego, że grupa ta jest coraz liczniejsza i żyje coraz dłużej.

Früher war das Fremdsprachenlernen unter den Senioren nicht so verbreitet, wie es heute der Fall ist. Ich bin der Meinung, dass dies die Konsequenz dessen ist, dass diese Gruppe immer zahlreicher ist und immer länger lebt.

\subsection{Voraussetzung des Lehrerberufs}

Beachtenswert sind die Aussagen, die in 5 Fragebögen zu finden waren. Sie betreffen die Spezifik des Lehrerberufes. Die Umfrageteilnehmer heben die Tatsache hervor, dass zu den Lehrerpflichten ständige Entwicklung gehört. Jeder Lehrer sollte außerdem den neusten Tendenzen in der Didaktik und Methodik folgen. Darüber hinaus ist die Bereitschaft mit verschiedenen Altersgruppen zu arbeiten von großer Bedeutung.

Osoby starsze chcą uczyć się języków obcych, a my, jako nauczyciele, powinniśmy być w stanie nauczać różne grupy.

Ältere Personen möchten Fremdsprachen lernen und wir, als Lehrer, sollten imstande sein, verschiedene Gruppen zu unterrichten.

Die nächste Frage in dieser Fragengruppe bezieht sich auf die Motivation der Studenten an der vorgeschlagenen Veranstaltung teilzunehmen. Die Teilnahme war fakultativ und die Studenten konnten die Veranstaltung wählen, die sie besuchten. In dieser Frage kreuzten die Befragten die von ihnen gewählte Antwort oder mehrere Antworten an, zusätzlich konnten sie sich auch zu diesem Thema frei äußern. Die Ergebnisse sind der unter dargestellten Tabelle zu entnehmen. 
Motivation zur Teilnahme am Workshop

Neugier

Es fehlt an ähnlichen Veranstaltungen im Studienprogramm

Ich wollte mein geragogisches Wissen erweitern

Ich interessiere mich eher für Projekte und deswegen nehme ich an diesem Workshop teil

Ich wollte etwas neues lernen
Anzahl der Antworten

17

28

7

27

Die Studenten konnten im Fragebogen auch ihre eigenen Antworten und Motivationsvorschläge darstellen. 4 Befragte weisen darauf hin, dass trotz des steigenden Bedarfs an Kursen für Senioren es an Fortbildungsangeboten für angehende oder schon praktizierende Lehrer fehlt.

Nie znalazłam kursów metodycznych przygotowujących do pracy z seniorami w żadnej placówce szkoleniowej, dlatego warsztaty są dla mnie jedyną szansą zdobycia wiedzy na ten temat.

Ich habe entsprechende methodische Kurse, die zur Arbeit mit Senioren vorbereiten an keiner der Fortbildungseinrichtungen gefunden, deswegen ist dieser Workshop für mich die einzige Chance das Wissen zu diesem Thema zu gewinnen.

Alle Befragte haben ausdrücklich betont, dass die Elemente der fremdsprachlichen Glottodidaktik im Studienprogramm berücksichtigt sein sollten. Studenten haben auch zusätzlich kommentiert, dass die glottodidaktische Spezialisierung, die sie gewählt haben, sie zur Arbeit mit unterschiedlichen Altersgruppen, sowohl im theoretischen, als auch im praktischen Sinne vorbereiten sollte.

\section{DATENANALYSE}

Die im Rahmen des Fragebogens gesammelten Daten zeigen eindeutig darauf, dass die heutigen Studenten sich vor allem um ihre Chancen auf dem Arbeitsmarkt kümmern, deswegen liegt ihnen eine gute Vorbereitung für die zukünftige Arbeit am Herzen, was ihren Aussagen zu entnehmen ist. Darüber hinaus sind die Befragten sich der zunehmenden Rolle der älteren Personen im Kontext des Fremdsprachenlernens bewusst.

Die Befragten waren nicht darüber einig, was die Tatsache angeht, wer zur Seniorengruppe gehört. 10 Personen assoziierten Senioren mit dem Ausschalten aus dem beruflichen Leben, 2 Personen mit Erfahrung. Nur eine Antwort kennzeichnete eine eindeutig positive Einstellung zu älteren Personen. Die erhobenen Ergebnisse liefern Beweise dafür, dass für junge Perso- 
nen die ältere Generation unbekannt ist und trotzt des Bewusstseins, dass Senioren auch eine Lernergruppe sein können, fußt das Wissen angehender Lehrer eher auf allgemeinen Grundsätzen. Die Mehrheit der Workshopteilnehmer ist nicht imstande festzustellen, ob die Gruppe der Senioren zahlreich ist. Nur 2 Personen verbinden das höhere Alter mit einem aktiven Lebensstil, der ein Fundament für den Denkwandel liegt.

Fast alle Befragten (34 Personen) haben bisher keine didaktische Erfahrung mit Seniorengruppen gesammelt, was die Erklärung ihres fehlenden Wissens in diesem Bereich sein kann. Dazu äußern die Befragten selbst das Bedürfnis methodische Grundgesetze der Fremdsprachengeragogik kennen zu lernen, wozu sie bisher keine Gelegenheit hatten. Dies führt auch dazu, dass sie sich für die Arbeit mit dieser Altersgruppe nicht vorbereitet fühlen. Die Studenten drücken eindeutig den Willen aus, ihr Wissen um oben genannte Aspekte zu bereichern.

In den offenen Fragen, in denen Studenten über ihre Motivation zur Teilnahme am Workshop berichteten, spiegelte sich große Angst um ihre Arbeitsperspektiven wider und was damit verbunden war, ihre Bereitschaft etwas Neues zu lernen um eigene Chancen auf dem Arbeitsmarkt zu verbessern. Beachtenswert waren auch Äußerungen über Voraussetzungen des Lehrerberufs, der in der heutigen Welt nicht nur großer Flexibilität bedarf, sondern auch der Bereitschaft zum ständigen Lernen und Aufgeschlossenheit in Kontakten mit verschiedenen Altersgruppen.

\section{FAZIT}

Die heutigen Studenten sind sehr motiviert, sie erkennen die neuen Möglichkeiten, die die Welt ihnen anbietet und sind bereit sich an die wechselnden Arbeitsvoraussetzungen anzupassen. Dazu brauchen sie aber theoretische und praktische Vorbereitung. Senioren sind eine bedeutsame Lernergruppe, die mit der Zeit zahlreicher sein wird, deswegen wäre es angebracht gerade dieser bisher unerforschten Altersgruppe mehr Achtung auch bei Planung der Studienangebote (sogar im Rahmen zusätzlicher Veranstaltungen) zu schenken.

\section{LITERATURVERZEICHNIS}

Bausch, K.-R., Christ, H., Krumm, H.-J. (Hrsg.). 2002. Handbuch Fremdsprachenunterricht. Tübingen-Basel: Francke Verlag.

Becker, S., Veelken, L., Wallraven, K.P. (Hrsg.). 2000. Handbuch Altenbildung: Theorien und Konzepte für Gegenwart und Zukunft. Opladen: Leske \& Budrich. 
Bubolz-Lutz, E., Gösken, E., Kricheldorf, C., Schramek, R. (Hrsg.). 2010. Geragogik. Bildung und Lernen im Prozess des Alterns. Das Lehrbuch. Stuttgart: Kohlhammer.

Caspari, D., Helbig, B., Schmelter, L., 2003. Forschungsmethoden: Explorativ-interpretatives Forschen. In: Bausch, K.-R., Christ, H.,Krumm, H.-J. (Hrsg.). Handbuch Fremdsprachenunterricht. Tübingen-Basel: Francke Verlag, 499-505.

Keller, M., 2009. In meinem Badezimmer bin ich überwacht. In: Frankfurter Allgemeine Zeitung, abgerufen am 10.01.2013.

Kolland, F., 2010. Standortbestimmung der Geragogik. In: Miklas, H. (Hrsg.). Geragogik- eine Herausforderung der Zukunft. Wien-Krems: LIT Verlag, 9-25.

Miklas, H. (Hrsg.). 2010. Geragogik- eine Herausforderung der Zukunft. Wien-Krems: LIT.

Veelken, L., 2000. Geragogik: Das soziogerontologische Konzept. In: Becker, S., Veelken, L., Wallraven, K.P. (Hrsg). Handbuch Altenbildung: Theorien und Konzepte für Gegenwart und Zukunft. Opladen: Leske \& Budrich, 87-94.

Wiłkomirska, A. 2005. Ocena ksztatcenia nauczycieli. Warszawa: Instytut Spraw Publicznych. 
